\section{A narrow-minded master}

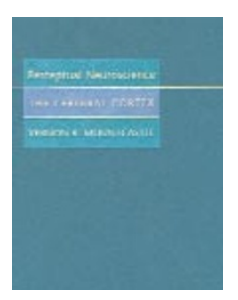

Perceptual Neuroscience: The Cerebral Cortex

by Vernon B. Mountcastle

Harvard University Press, Cambridge, Massachusetts, 1998. \$59.95

hardcover, pp 448

ISBN 0-674-66188-5

\section{Reviewed by Charles G. Gross}

Vernon B. Mountcastle is the doyen of systems neuroscience. For more than fifty years, he has produced a steady stream of influential textbooks, discoveries, ideas and young scientists. Early in his career, Mountcastle began a sustained attack on the somesthetic system from receptor to cortex and was one of the first to combine sophisticated psychophysical methods with singleneuron recording in monkeys. Then, at the conventional retirement age, he began pioneering studies of the properties of posterior parietal cortex in attention and spatial vision. Perhaps his major achievement was his discovery of the importance of the column to cerebral cortex organization.

Perceptual Neuroscience: The Cerebral Cortex is a superb, if rather selective, survey of the phylogeny, ontogeny, anatomy, physiology and organization of the cerebral cortex. Emphasizing the role of the cortex in perceptual processing, this well-written, well-produced volume draws on over 1100 citations. What is particularly valuable about this book is the insights it provides into the thinking of one of our most active senior neuroscientists. How does he see contemporary neuroscience in the context of its history? What are the most important recent findings and problems? Where is the field going? Of even more interest, where does he think the field should go? On all these questions, this book provides an idiosyncratic personal view.

The first idiosyncracy is the lacunae. Virtually nobody is mentioned from before Mountcastle's student days: no Fritsch and Hitzig, no Ferrier, no Jackson, no Adrian (except on EEG), no Lashley (except on cytoarchitectonics). In contrast to Newton, there is no standing on shoulders. There are several long chapters on development, but no mention of David Hubel and Torsten Wiesel's work on monocular experience and ocular dominance columns, which exploded into the exciting field of activity-dependent neurodevelopment. There is a chapter

Charles Gross is at the Psychology Dept., Princeton University, Princeton, New Jersey 08540, USA.

e-mail:cggross@phoenix.princeton.edu on synaptic plasticity in the hippocampus, but the recent cascade of papers (such as Elizabeth Gould's) showing structural plasticity in the hippocampus is ignored. Equally striking is the failure to cite the extraordinary findings of Michael Merzenich, Jon Kaas and others on changes in cortical organization with peripheral lesions or sensory experience. Sperry's work on hemispheric laterality is ignored, and brain imaging gets a few sentences.

The book champions "Perceptual neuroscience: the union of systems neurophysiology and psychophysics" and its central method, the recording of isolated neurons during performance by trained monkeys. Yet the clearest demonstration of a close relation between single-neuron activity and perception, namely William Newsome's elegant demonstration of the perceptual effects of stimulation in an MT column, is nowhere to be found.

The primary method for studying cortical function, indeed virtually the sole method until halfway through this century, has been the study of brain-injured humans and experimental lesions in animals. However, Mountcastle hardly mentions this extensive body of work. Yet it was such lesion studies that led directly to the singleneuron studies of cortical function of which Mountcastle does approve. One example is my own studies of the properties of inferior temporal cortex neurons (which Mountcastle describes both generously and accurately). Another example is Mountcastle's work on posterior parietal neurons, which, from his earlier account, seemed influenced by human lesion studies.

Mountcastle's book is idiosyncratic not only in what it omits but in what it attacks and what it advocates. Modern cortical physiology may be said to have begun with Hubel and Wiesel's scheme for the hierarchical processing of visual information from the retina through the hypercomplex cells of striate cortex. In the primate, this simple suggestion has now been expanded into two streams of visual processing that extend from striate cortex through visual areas in the temporal and parietal lobes on into pre- frontal cortex. The ventral stream emphasizes form and color, and the dorsal stream, space and movement. Each of the streams has several substreams that separate and rejoin, converging and diverging. For example, separate substreams for motion and space may be discerned within the dorsal stream, and for form and color within the ventral stream. Even the major streams are repeatedly crosslinked, particularly in areas MT and STP, and both streams seem to converge in the hippocampus and in prefrontal cells. It is unclear how the streams bind into unified perceptions, what the role of the subcortical connections is, how attention modifies stimulus processing and so on. Even the nicknames 'what' and 'where' for the dorsal and ventral streams have been usefully challenged. Despite these and other questions, this concept of sequential processing has been an enormously powerful heuristic device for studying the organization of the primate visual cortices. Furthermore, it is now being expanded to other sensory systems and infraprimate species.

Yet, this 'two visual streams' idea and its originators, Mortimer Mishkin and Leslie Ungerleider, are nowhere mentioned in the text. Instead, Mountcastle sets up a simple straw hierarchy of a pyramidal shape leading to a single superordinate peak and shows, convincingly, its inadequacies. In place of hierarchical processing, Mountcastle gives us "coupling between nodes," described as "reentry," defined as "a process of parallel and recursive signaling along ordered anatomical connections that achieve integration by giving rise to constructive properties within and between [neural] maps," citing Gerald Edelman and colleagues. Mountcastle then admits "what those constructive and correlative properties are and how they arise remain unclear." Despite this understandable puzzlement, Mountcastle persists in using "re-entry" as a magic wand to explain cortical function.

In a revealing epilogue, the author gives us his prescription for studying cortical function. Take "1000 or so microelectrodes implanted to cover the several nodes of the parieto-frontal distributed system as a monkey reaches to a target....and later analysis of several hundred action potentials during each trial on each of the 1,000 channels. Such an experiment will mark the transition of perceptual neuroscience from little science (one or a few investigators huddled about a minicomputer) to big science with experiments executed and the results analyzed by an accomplished team of scientists.... Presently no other experimental path is obvious." When that time comes, I'll confine myself to book reviews. 